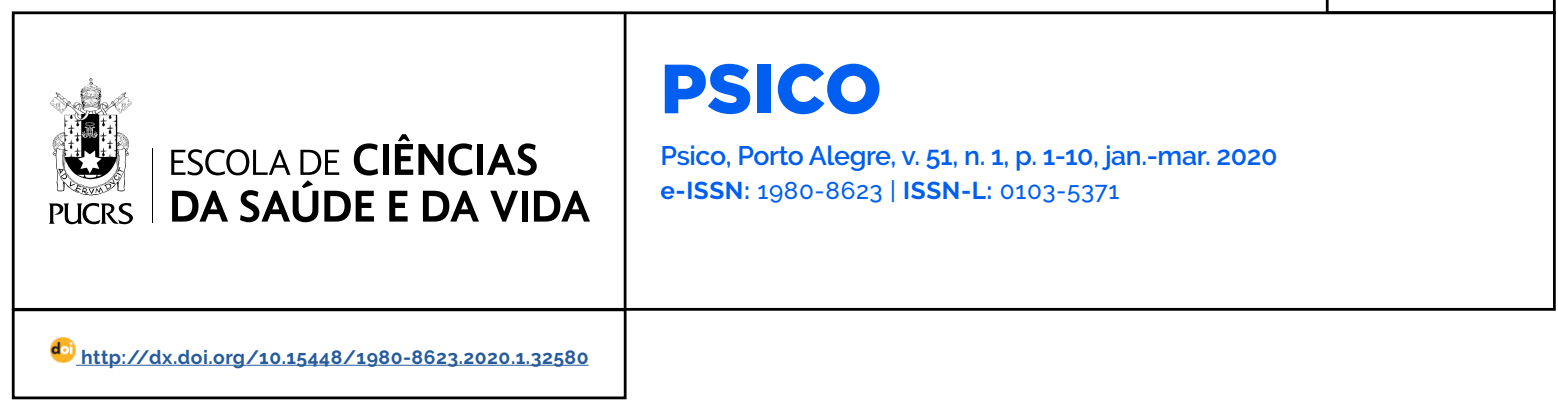

ARTIGOS

\title{
Escala de Gerenciamento da Impressão e Autoengano - IPIP: um teste de diferentes modelos teóricos
}

\author{
Impression Management and Self-Deceptive Scales - IPIP: a test of different theoretical \\ models \\ Escala de Gestión de la Impresión y Autoengaño-IPIP: una prueba de diferentes \\ modelos teóricos
}

\begin{abstract}
Ariela Raissa Lima Costa ${ }^{1}$
orcid.org/0000-0002-5942-6466 arielalima10@gmail.com
\end{abstract}

\section{Nelson Hauck Filho'}

orcid.org/0000-0003-0121-7079

hauck.nf@gmail.com

Recebido em: 21 nov. 2018.

Aprovado em: 15 ago. 2019.

Publicado em: XX XXX. 2020.

\section{(c) (1)}

Artigo está licenciado sob forma de uma licença Creative Commons Atribuição 4.0 Internacional.
Resumo: Um desafio da avaliação psicológica via autorrelato são vieses de resposta, isto é, formas de responder que mascaram a verdadeira medida do traço latente do indivíduo. A desejabilidade social ocorre quando a pessoa fornece uma avaliação excessivamente positiva de si, baseado em valores sociais. Na literatura, destacam-se três modelos teóricos da desejabilidade social, em que são sugeridas estruturas de dois e três fatores, além de uma estrutura com dois fatores e quatro facetas (ou modelo de quatro fatores). Este trabalho se propõe a explorar diferentes modelos fatoriais para a Escala de Gerenciamento da Impressão e de Autoengano-IPIP. A amostra foi de 466 adultos ( $M=25,22$ anos, $D P$ $=7,05,60,27 \%$ mulheres). O modelo de quatro fatores apresentou o melhor ajuste aos dados, mas o modelo original de dois fatores produziu a solução fatorial mais simples e clara. Implicações dos achados do presente estudo quanto ao cômputo de escores e interpretação do instrumento são discutidas.

Palavras-chaves: viés egoista, viés moralista, desejabilidade social, viés de resposta.

Abstract: One challenge of self-report assessments is response styles, individual preferences for some of the options in a given response scale that might bias the true latent trait level of the respondent. Socially desirable responding consists of one individual making efforts to display oneself in a favorable manner given perceived social values. Three factor models of social desirability have received attention, with two and three factors, and the last one two factors and four facets (or four-factor model). In the present study, we explore the extent to which these three factor models represent the structure of the Impression Management and Self-Deception Scale-IPIP. Participants were 466 adults ( $M=25.22$ years, $D P=7.05,60.27 \%$ females). The four-factor model yielded the best fit to the data, but the original two-factor structure resulted in the simpler and clearer factor solution. Implications of these findings as to the scoring and interpretation of the instrument are discussed. Keywords: egoistic bias, moralistic bias, social desirability, response bias

Resumen: Uno reto de la evaluación psicológica por el autoinforme, son los sesgos de respuesta, es decir, la manera de responder que enmascaran la verdadera medida del rasgo latente individual. La deseabilidad social ocurre cuando la persona proporciona una evaluación excesivamente positiva de sí basado en valores sociales. Tres modelos factoriales de la deseabilidad social se destacan en la literatura, con dos y tres factores y, lo último con dos factores y cuatro facetas (o modelo de cuatro fatores). En este estudio, exploramos lo cuanto cada modelo factorial representa la estructura de la Escala de Gestión de la Impresión y Autoengaño-IPIP. La muestra fue de 466 adultos ( $M=25,22$ años, $D P=7,05,60,27 \%$ mujeres). El modelo de cuatro factores tuvo lo mejor ajuste a los datos, pero el modelo original de dos factores fue la solución mas simple y clara. Implicaciones de estos hallados para la puntuación e interpretación del instrumento son discutidos. Palabras claves: sesgo egoista, sesgo moralista, deseabilidad social, sesgo de respuesta. 
Interações sociais são baseadas na forma como cada um se comporta e é percebido pelo outro, isto é, as pessoas se influenciam e são influenciadas pelo meio social. Alguns contextos aumentam a percepção que a pessoa tem sobre o próprio comportamento, diminuindo a espontaneidade e aumentando o controle na manifestação de comportamentos especíicos, seja para causar uma boa impressão ou, pelo menos, evitar uma má impressão. As normas sociais, o desejo e a capacidade de manipulação do sujeito determinam quais comportamentos controlar e como fazê-lo. Esse manejo comportamental ocorre em nivel não verbal (e.g., gestos, expressões faciais) e verbal (e.g., comunicação oral e escrita) (Leary \& Bolino, 2017). $\mathrm{Na}$ avaliação psicológica, isso ocorre, por exemplo, quando os sujeitos respondem a instrumentos de autorrelato tentando passar uma imagem positiva da sua personalidade - sendo, a isso, dado o nome de respostas socialmente desejáveis.

Respostas socialmente desejáveis figuram como um dos principais vieses de respostas a instrumentos de autorrelato. Esse viés se caracteriza por a pessoa fornecer respostas excessivamente positivas a itens de autorrelato, sendo que essa manipulação vai depender das características individuais, das características dos itens e do contexto de avaliação (Ziegler, 2015). A desejabilidade social relaciona-se à forma como o indivíduo se comporta no meio social, sendo que as pessoas que fornecem respostas mais distorcidas costumam se apresentar como muito sociáveis e buscam agradar aos outros, seja para alcançar um objetivo pessoal ou para se adaptar ao grupo (Malham \& Saucier, 2016). A manipulação das respostas (ou dos comportamentos) pode ocorrer de forma pensada, em que a pessoa sabe que a sua resposta (ou comportamento) não condiz com o seu traço latente. Ainda, essa manipulação pode ocorrer de forma não consciente, de modo que a pessoa responde de forma socialmente desejável por acreditar que possui tais caracteristicas positivas, ignorando os seus traços menos desejáveis (Schwardmann \& van der Weele, 2016).

Os valores morais também influenciam na desejabilidade social (Trapnell \& Paulhus, 2012). Pessoas que possuem valores individualistas são mais prováveis de se apresentarem como individuos superdotados em capacidades cognitivas e físicas. Em contraste, individuos com valores coletivos tendem a se voltar para o outro e a se apresentar como superdotados em capacidades sociais e afetivas (Paulhus, 2002; Paulhus \& John, 1998). Devido às múltiplas influências sob as respostas socialmente desejáveis, alguns modelos teóricos foram formulados, que podem variar quanto ao número de dimensões que atribuem à desejabilidade social (Paulhus, 2002). O presente estudo, além de apresentar uma análise psicométrica de um instrumento que avalia desejabilidade social - a Escala de Gerenciamento da Impressão de Autoengano -, busca contribuir para uma melhor compreensão do fenômeno, ao analisar a plausibilidade desses diferentes modelos.

Existem três modelos proeminentes que explicam a estrutura fatorial da desejabilidade social: dois (Paulhus, 1984), três (Paulhus \& Reid, 1991) e dois fatores e quatro facetas (ou modelo de quatro fatores) (Paulhus \& John, 1998). O primeiro modelo discrimina dois tipos de respostas socialmente desejáveis, com base no tipo de processamento, que pode ser consciente ou inconsciente, dando origem aos fatores gerenciamento da impressão e autoengano, respectivamente. Assim, um individuo que intencionalmente realça os seus atributos positivos tende a possuir altos niveis de gerenciamento da impressão, enquanto outro que tem a crença internalizada de que é superior tende a pontuar alto em autoengano.

O segundo modelo considera, além do tipo de processamento, o tipo da tática utilizada, que pode se dar a partir do aprimoramento ou da negação (Paulhus \& Reid, 1991). Nesse caso, o manejo da impressão sempre envolve o realce de habilidades, enquanto o autoengano pode se dar a partir da crença irreal nas próprias virtudes ou da falta de percepção de defeitos. Desse modo, as dimensões possiveis são o gerenciamento da impressão, o autoengano positivo e o autoengano negativo. 
Por sua vez, a terceira perspectiva (Paulhus \& John, 1998) foca no entendimento dos motivos subjacentes à desejabilidade social. A saber, o modelo separa o fenômeno entre os valores individuais e coletivos, e propõe uma solução de dois fatores, chamados de viés egoísta e moralista. Cada um desses vieses pode se manifestar por meio de dois processos, um de tipo consciente e outro de tipo não consciente. Assim, o viés egoísta ocorre em pessoas que se acham excepcionalmente talentosas e inteligentes, que tendem a se mostrar ou perceber como "super-heróis". As facetas dessa dimensão ampla são manejo do eu (consciente) e autoengano positivo (não consciente). O viés moralista pode ser observado em pessoas que se consideram um modelo de bom cidadão, de defensor da moral e dos bons costumes, que tendem a se mostrar ou perceber como "santos". As facetas dessa dimensão ampla são manejo da atuação social (consciente) e autoengano negativo (não consciente).

Os três modelos podem ser compreendidos de forma complementar. Enquanto Paulhus (1984) e Paulhus e Reid (1991) abordam os componentes cognitivos e contextuais da ação, Paulhus e John (1998) descrevem valores e motivos básicos dos comportamentos. Em sintese, para que respostas socialmente desejáveis ocorram, são necessários três elementos: 1) o sujeito perceba que tem a capacidade cognitiva para alterar suas respostas; 2) o contexto indique a ele que é necessário uma alteração das suas respostas; e 3) o individuo considere moralmente aceitável realizar essa alteração em suas respostas para alcançar um objetivo intrínseco à testagem (Ellingson, 2012). Todos os modelos consideram que parte das respostas distorcidas são diretamente controladas pelo sujeito (i.e., gerenciamento da impressão e suas facetas, manejo do eu e manejo da atuação social) e parte não são óbvias ao próprio sujeito, que filtra, sem perceber, as informações sobre as próprias características (i.e., autoengano e suas facetas, autoengano positivo e autoengano negativo).

Essas abordagens teóricas produziram diversos instrumentos para avaliar desejabilidade social. O mais utilizado é a Escala de Desejabilidade Social de
Marlow-Crowne (MCSDS; Crowne \& Marlowe, 1960) que avalia apenas comportamentos socialmente exagerados relacionados ao gerenciamento da impressão (e.g. "Estou sempre disposto a admitir quando cometo um erro"), não contemplando os demais aspectos da desejabilidade. Uma alternativa à MCSDS é o Inventário Balanceado de Respostas Desejáveis (Balanced Inventory of Desirable Responding ou BIDR; Paulhus, 1984). Esse instrumento avalia a desejabilidade social de uma perspectiva mais ampla e voltada para nuances mais sutis da desejabilidade. O BIDR possui uma versão com 40 itens (baseada na estrutura de dois fatores) e outra com 60 itens (baseada na estrutura de três fatores). Uma versão estendida dessa ferramenta, com 80 itens, foi elaborada de modo a contemplar o modelo de dois fatores e as suas quatro facetas (Paulhus, 2005).

Embora o escopo teórico seja importante, o número de itens acaba sendo também um aspecto levado em consideração na escolha por uma escala para avaliar desejabilidade social. Por isso, uma versão reduzida do BIDR foi desenvolvida a partir da base internacional de itens International Personality Item Pool (IPIP). Esse instrumento foi nomeado de Escala de Gerenciamento da Impressão e Autoengano IPIP, contém 30 itens que avaliam o modelo de dois fatores da desejabilidade social, sendo 20 itens para gerenciamento da impressão, e 10 itens para autoengano.

Embora a Escala de Gerenciamento da Impressão e Autoengano - IPIP tenha sido elaborada com base no modelo de dois fatores, isso não significa que os demais modelos não sejam estruturas plausíveis. Como explicitado antes, os modelos de três e de quatro fatores são teoricamente aninhados à abordagem de dois fatores, consistindo apenas em um aprofundamento dos diferentes processos envolvidos em cada uma dessas dimensões amplas. É possivel, dessa forma, que a escala contenha itens que contemplem variância única relacionada aos componentes mais específicos desses modelos. Por exemplo, se alguns dos itens de gerenciamento da impressão abordam 
aspectos conscientes desse fenômeno enquanto outros endereçam processos menos conscientes, então talvez seja possivel identificar as duas facetas dessa dimensão ampla, tal como propostas por Paulhus e John (1998). Essa investigação de diferentes modelos candidatos poderia oferecer uma diretriz a pesquisadores sobre quais itens poderiam ser utilizados para compor um escore agregado de cada faceta da desejabilidade social. Por isso, o presente estudo teve por objetivo o teste de diferentes modelos teóricos de dois, três e quatro fatores para a Escala de Gerenciamento da Impressão e Autoengano - IPIP.

\section{Método}

\section{Participantes}

Os participantes do estudo foram 466 adultos brasileiros com idades entre 18 e 47 anos $(M=$ 25,22 anos; $D P=7,05$ ) sendo 60,27\% mulheres $49,6 \%$ solteiros, $96,3 \%$ com ensino superior incompleto,73,1\% brancos e 20,1\% pardos. As coletas foram feitas por meio de um questionário online e presencial, após autorização pelo comitê de Ética da Universidade São Francisco sob CAAE 91793718.7.0000.5514.

\section{Instrumentos}

Escala de Gerenciamento da Impressão e Autoengano do IPIP (Impression Management and Self-Deception Scales - IPIP; IMSDS-IPIP). O instrumento é de autorrelato e possui 30 itens que avaliam a desejabilidade social em duas dimensões, gerenciamento da impressão e autoengano. A escala de resposta dos itens é do tipo Likert, 1 = Discordo totalmente e 5 = Concordo totalmente. A versão completa do instrumento pode ser localizada no Anexo 1. O site do IPIP (http//www.ipip.ori.org, recuperado em 14, janeiro, 2020), informa que a consistência interna dessas escalas foi estimada em 0,82 (gerenciamento da impressão) e 0,80 (autoengano). Além disso, a correlação da versão IPIP com o instrumento original foi 0,79 para as dimensões de gerenciamento da impressão, e 0,73 para as dimensões de autoengano.

\section{Procedimentos de coleta de dados}

O projeto foi previamente submetido a um Comitê de Ética, em concordância com Resolução n. ${ }^{\circ}$ 196/96 do Conselho Nacional de Saúde (CAAE: 53659515.2.0000.5514) do Brasil. A coleta ocorreu em uma instituição de ensino superior do estado de São Paulo. Os diretores dos campi autorizaram a coleta e os coordenadores dos cursos indicaram em quais turmas poderia acontecer a aplicação da pesquisa. Na coleta online, o participante acessou um link do site SurveyMonkey, em que era apresentada uma breve explicação dos objetivos da pesquisa, seguida pelo Termo de Consentimento Livre e Esclarecido (TCLE) e, então, pelos testes. Na coleta presencial, foi explicado verbalmente o objetivo da pesquisa, bem como a importância científica da mesma. Em seguida, o TCLE foi lido e assinado, quando então se apresentaram os instrumentos para que os participantes respondessem.

\section{Análise dos dados}

O presente estudo consistiu no teste de modelos candidatos para o instrumento a partir de uma abordagem exploratória. Em primeiro lugar, a análise fatorial exploratória parece mais adequada à presente situação por ser desconhecida a distribuição de itens em cada possivel faceta da desejabilidade social. Em segundo lugar, itens que avaliam aspectos de diferenças individuais tendem a apresentar carga cruzada, tendendo a ser implausiveis modelos que assumem a inexistência dessas cargas cruzadas (Asparouhov \& Muthén, 2009).

A dimensionalidade do instrumento foi investigada com o método Análise Paralela com permutação de dados, capaz de estimar as comunalidades entre os itens e minimizar a variância não explicada a partir da determinação de um número de fatores (Timmerman \& LorenzoSeva, 2011). A análise foi conduzida com o software Factor 10.3 (Lorenzo-Seva \& Ferrando, 2015). Foi empregado um estimador robusto, - Weighted Least Squares Mean and Variance adjusted (WLSMV) com correlações policóricas. Esse estimador foi escolhido pelo fato de que 
as escalas Likert utilizadas são compostas por categorias ordenadas em vez de variáveis continuas (Zumbo, Gadermann, \& Zeisser, 2007). Ainda foram calculados os indices de ajuste quiquadrado, Root Mean Square Adjusted (RMSEA), Confirmatory Fit Index (CFI) e Tucker-Lewis Index (TLI). Essas análises foram conduzidas no programa Mplus 8.1.

\section{Resultados}

Os dados se mostraram adequado para análise fatorial exploratória ordinal, $\mathrm{KMO}=0,83$, teste de esfericidade de Bartlett $=3483,1 ; p<0,001$, indicando que a matriz de correlação se distancia de uma matriz identidade. Foram testados três modelos candidatos: dois (Paulhus, 1984), três (Paulhus \& Reid, 1991) e quatro fatores (Paulhus \& John, 1998). A análise paralela indicou um melhor ajuste aos dados, o que foi também sustentado pelos indices de ajuste obtidos para cada modelo. Os resultados estão apresentados na Tabela 1.

\section{TABELA 1}

\begin{tabular}{lccccc}
\hline & $\boldsymbol{x}^{\mathbf{2}}$ & RMSEA & CFI & TLI & $\boldsymbol{\Delta} \boldsymbol{\chi}^{\mathbf{2}}$ \\
\hline $\mathbf{2}$ fatores & $1785,952^{*}$ & 0,073 & 0,802 & 0,771 \\
$\mathbf{3}$ fatores & $1187,330^{*}$ & 0,058 & 0,882 & 0,852 & $456.391^{*}$ \\
$\mathbf{4}$ fatores & $917,763^{*}$ & 0,051 & 0,916 & 0,886 & $231.440^{*}$ \\
$\mathbf{4}$ fatores com correlações residuais & $768,685^{*}$ & 0,045 & 0,937 & 0,913 & $149.078^{*}$ \\
\hline
\end{tabular}

Indices de Ajustes para Diferentes Estruturas Fatoriais da IMSD-IPIP

Nota. ${ }^{*} p<0,001$

Apesar de a solução de quatro fatores ter mostrado o melhor ajuste, uma análise teórica também garantiu a interpretabilidade da solução de dois fatores, aquela que inspirou o desenvolvimento da escala (ver Tabela 2). Na solução de dois fatores, foram identificados os fatores gerenciamento da impressão $(\alpha=0,83)$ e autoengano $(\alpha=0,77)$. Como esperado, $a$ correlação entre os fatores foi negativa $(r=-0,33$, $p<0,05)$, indicando que individuos que pontuam mais em um estilo de autoapresentação enganosa inconsciente tendem a se engajar menos em esforços mais conscientes de gerenciamento da impressão e vice-versa.

A solução de três fatores apresentou uma boa interpretabilidade. Os itens da dimensão gerenciamento da impressão (itens 1 a 20) se mantiveram conforme o modelo de dois fatores. Quanto aos itens da dimensão autoengano positivo (itens 21 a 25), verificou-se que os itens 24 e 25 . apesar de terem a sua carga fatorial primária no fator autoengano positivo, também apresentaram cargas cruzadas na dimensão interpretada como autoengano negativo, como apresentado na Tabela 2. Por sua vez, os itens sobre autoengano negativo (itens 26 a 30) carregaram em um fator à parte. Os coeficientes de consistência interna das dimensões autoengano positivo e negativo foram, respectivamente, 0,68 e 0,67. O coeficiente da dimensão gerenciamento da impressão se manteve o mesmo da solução de dois fatores 0,83 . A correlação entre os fatores foi de $r_{\text {autoengano }}$ positivo $\times$ autoengano negativo $=0,00(p>0,05), r_{\text {gerenciamento da }}$ impressão $\times$ autoengano positivo $=-0,16(p<0,05), r_{\text {gerenciamento }}$ da impressão $\times$ autoengano negativo $=0,36(p<0,05)$.

Como hipotetizado, os quatro fatores se mostraram coerentes com o panorama teórico do modelo de Paulhus e John (1998). Mais especificamente, o fator 1 esteve mais relacionado a aspectos conscientes e morais da desejabilidade social, ou seja, manejo da atuação social ("Devolvo o troco extra quando o caixa comete um erro"). Enquanto isso, o fator 2 explicou aspectos inconscientes, mas de tipo egoísta, ou seja, autoengano positivo ("Sei que minhas decisões estão corretas"). O fator 3 parece 
se conectar mais a aspectos inconscientes, mas dentro da esfera moral, ou seja, autoengano negativo ("Nem sempre sou o que pareço ser"). Por sua vez, o fator 4 esteve associado a aspectos conscientes e egoístas da desejabilidade social, ou seja, o manejo do eu ("Falo palavrões"). Esses fatores apresentaram, respectivamente, coeficientes de confiabilidade de, 0,79, 0,68, 0,67 e 0,60 . As cargas fatoriais dos itens nos quatro fatores podem ser observadas na Tabela 2.

TABELA 2 - Cargas Fatoriais para Solução de Dois e Quatro Fatores do IMSDS-IPIP

\begin{tabular}{|c|c|c|c|c|c|c|c|c|c|c|}
\hline \multirow{2}{*}{ Itens } & \multirow{2}{*}{$\begin{array}{l}\text { Aspecto } \\
\text { teórico }\end{array}$} & \multicolumn{2}{|c|}{ Dois fatores } & \multicolumn{3}{|c|}{ Três fatores } & \multicolumn{4}{|c|}{ Quatro fatores } \\
\hline & & F1 & $\mathbf{F 2}$ & F1 & $\mathbf{F 2}$ & F3 & F1 & $\mathbf{F 2}$ & F3 & F4 \\
\hline $\begin{array}{l}1 \text { Jamais pegaria coisas } \\
\text { que não são minhas. }\end{array}$ & $\mathrm{Gl}$ & $-0,54$ & 0,03 & $-0,58$ & 0,10 & 0,06 & 0,60 & 0,20 & 0,04 & 0,06 \\
\hline 2 Jamais sonegaria impostos. & $\mathrm{Gl}$ & $-0,55$ & $-0,09$ & $-0,59$ & 0,21 & 0,01 & 0,57 & 0,11 & 0,13 & $-0,01$ \\
\hline $\begin{array}{l}3 \text { Acredito que nunca há } \\
\text { uma desculpa para mentir. }\end{array}$ & Gl & $-0,50$ & 0,12 & $-0,55$ & 0,03 & 0,13 & 0,42 & 0,20 & $-0,01$ & $-0,19$ \\
\hline $\begin{array}{l}4 \text { Sempre admito quando } \\
\text { cometo um engano. }\end{array}$ & $\mathrm{Gl}$ & $-0,41$ & 0,17 & $-0,45$ & $-0,05$ & 0,12 & 0,45 & 0,24 & $-0,07$ & 0,03 \\
\hline 5 Raramente falo sobre sexo. & $\mathrm{Gl}$ & $-0,37$ & $-0,21$ & $-0,42$ & 0,38 & 0,09 & 0,23 & 0,05 & 0,35 & $-0,31$ \\
\hline $\begin{array}{l}6 \text { Devolvo o troco extra } \\
\text { quando o caixa comete } \\
\text { um erro. }\end{array}$ & $\mathrm{Gl}$ & $-0,48$ & 0,04 & $-0,50$ & 0,04 & 0,00 & 0,51 & 0,12 & $-0,02$ & 0,03 \\
\hline 7 Tento seguir as regras. & $\mathrm{Gl}$ & $-0,53$ & 0,02 & $-0,58$ & 0,14 & 0,11 & 0,49 & 0,18 & 0,10 & $-0,15$ \\
\hline $\begin{array}{l}8 \text { Facilmente resisto a } \\
\text { tentações. }\end{array}$ & $\mathrm{Gl}$ & $-0,26$ & 0,13 & $-0,29$ & $-0,04$ & 0,12 & 0,16 & 0,13 & $-0,04$ & $-0,20$ \\
\hline 9 Falo a verdade. & Gl & $-0,59$ & 0,17 & $-0,64$ & $-0,01$ & 0,15 & 0,61 & 0,29 & $-0,03$ & $-0,04$ \\
\hline $\begin{array}{l}10 \text { Raramente cometo } \\
\text { excessos prazerosos. }\end{array}$ & $\mathrm{Gl}$ & $-0,45$ & $-0,02$ & $-0,49$ & 0,17 & 0,11 & 0,33 & 0,12 & 0,13 & $-0,27$ \\
\hline $\begin{array}{l}11 \text { Algumas vezes, tive } \\
\text { que mentir. }\end{array}$ & $\mathrm{Gl}$ & 0,30 & $-0,13$ & 0,31 & 0,10 & $-0,01$ & $-0,04$ & 0,02 & 0,14 & 0,42 \\
\hline 12 Falo palavrões. & Gl & 0,48 & 0,06 & 0,51 & $-0,13$ & 0,05 & $-0,23$ & 0,08 & $-0,03$ & 0,44 \\
\hline 13 Bajulo para me dar bem. & $\mathrm{Gl}$ & 0,52 & 0,01 & 0,52 & 0,03 & 0,20 & $-0,53$ & 0,07 & 0,17 & $-0,04$ \\
\hline $\begin{array}{l}14 \text { Nem sempre sou o que } \\
\text { pareço ser. }\end{array}$ & $\mathrm{Gl}$ & 0,39 & $-0,25$ & 0,41 & 0,24 & 0,01 & $-0,25$ & $-0,05$ & 0,30 & 0,24 \\
\hline 15 Quebro regras. & $\mathrm{Gl}$ & 0,65 & 0,09 & 0,68 & $-0,19$ & 0,05 & $-0,41$ & 0,05 & $-0,07$ & 0,44 \\
\hline $\begin{array}{l}16 \text { Trapaceio para } \\
\text { me dar bem. }\end{array}$ & $\mathrm{Gl}$ & 0,70 & $-0,02$ & 0,71 & 0,04 & 0,21 & $-0,71$ & 0,04 & 0,20 & $-0,05$ \\
\hline
\end{tabular}




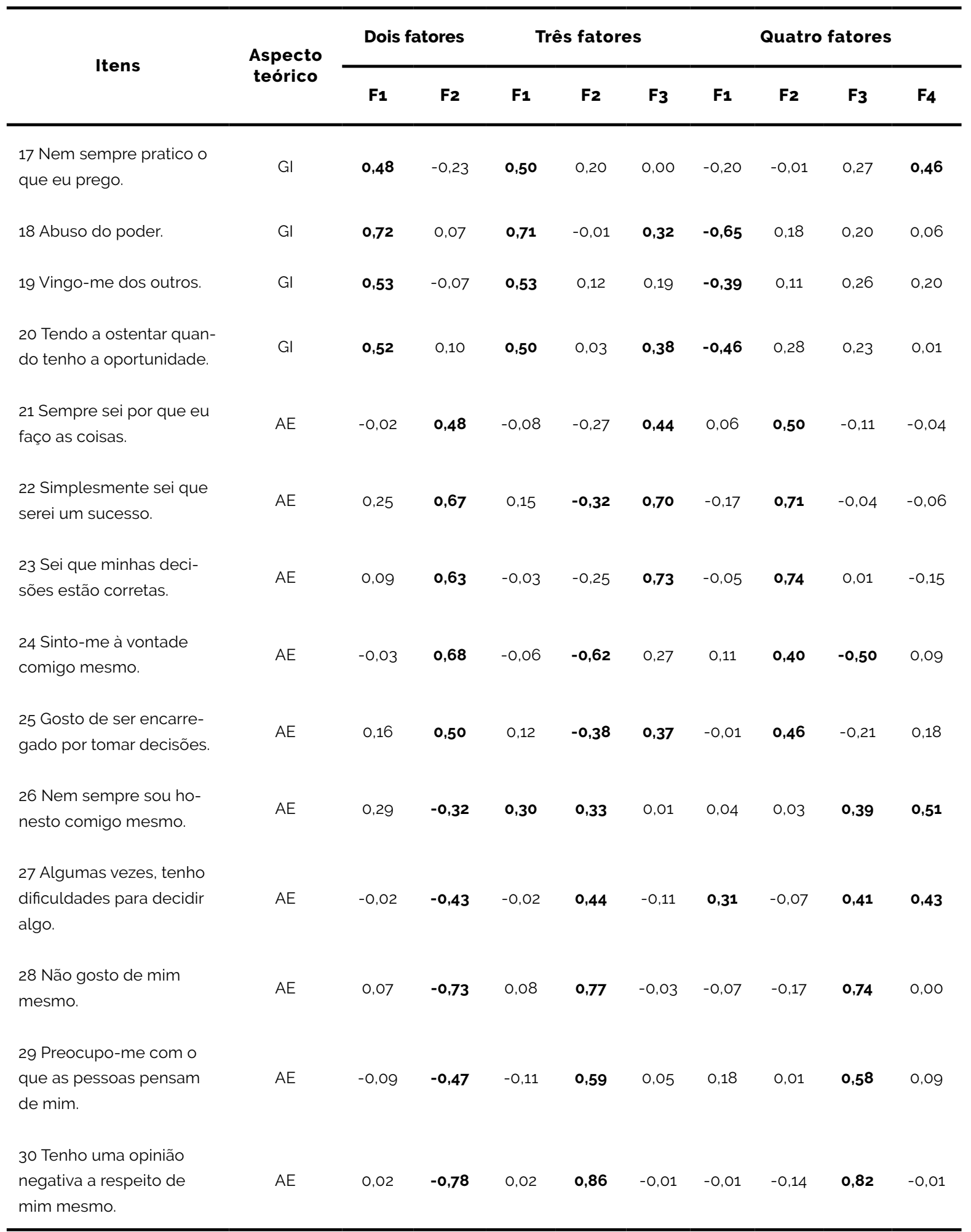

Nota. $\mathrm{Gl}$ = gerenciamento da impressão; $\mathrm{AE}$ = autoengano.

O melhor ajuste, não obstante, ocorreu para um modelo de quatro fatores correlacionados, como visto na Tabela 1. Da mesma forma como os modelos de dois e de três fatores, esse modelo se mostrou interpretável de acordo com a perspectiva teórica de dois fatores e quatro facetas de Paulhus e John (1998). Nessa perspectiva, dois fatores chamados viés egoista e viés moralista se subdividem nas facetas de manejo do eu, autoengano negativo, manejo da atuação social e autoengano positivo. A solução de quatro fatores encontrada foi coerente com 
esse modelo de facetas, como se pode ver a partir das cargas fatoriais exibidas na Tabela 2. As correlações entre esses fatores foram: $r_{\text {gerenciamento }}$ do eu $\times$ da atuação social $=-0,32(p<0,05), r_{\text {gerenciamento do eu }}$ $\times$ autoengano positivo $=-0,17(p<0,05), r_{\text {gerenciamento do eu } \times}$ autoengano negativo $=0,08(p>0,05), r_{\text {gerenciamento da atuação }}$ social $\times$ autoengano positivo $=0,04(p>0,05), r_{\text {gerenciamento da }}$ atuação social x autoengano negativo $=-0,22(p<0,05), r_{\text {autoengano }}$ positivo $\times$ autoengano negativo $=-0,21(p<0,05)$. Mesmo tendo sido o modelo com o melhor ajuste, os índices se mostraram um tanto empobrecidos, motivo pelo qual se buscou determinar se a falta de ajuste se deveria à presença de correlações residuais não modeladas. De fato, ao especificar correlações residuais entre alguns itens com conteúdo similar e correlação moderada $\left(r_{\text {item1 xitem } 2}=0,47, r_{\text {item } 22 \times \text { item }}\right.$ ${ }_{23}=0,57, r_{\text {item } 28 \times \text { item } 30}=0,61, p<0,01$ ), o ajuste se mostrou bastante razoável.

\section{Discussão}

O objetivo deste estudo foi o teste de diferentes modelos teóricos de dois, três e quatro fatores para a Escala de Gerenciamento da Impressão e Autoengano - IPIP. De fato, as análises sustentaram a estrutura original de dois fatores do instrumento. Duas dimensões amplas se mostraram interpretáveis como autoengano positivo (self-deception enhancement) e gerenciamento da impressão (impression management), com coeficientes de consistência interna similares à versão sueca ( $\alpha=0,82$ para as duas subescalas) (Bäckström, 2007). Os resultados indicaram que é empiricamente admissivel o uso do instrumento em sua forma original, com dois fatores, além do uso de três fatores, e de forma cuidadosa, é possivel o uso dos quatro fatores.

Na maioria dos casos, os itens do instrumento apresentaram cargas fatoriais nos fatores teoricamente esperados. As exceções foram os itens 5 ("Raramente falo sobre sexo") e 8 ("Facilmente resisto a tentações"). O item 5 obteve cargas cruzadas em ambos os modelos. Isso pode ter ocorrido por abordar questões sexuais, o que a pessoa teria dificuldade em admitir e negar, pois gera uma dúvida de qual o tipo de impressão que estaria dando ao outro (Leary \&
Bolino, 2017), e, também, por ser considerado um tema tabu, faz com que variados mecanismos de defesa e autoproteção do indivíduo sejam ativados (Vecchione, Alessandri, \& Barbaranelli, 2013). Por sua vez, o item 8 apresentou carga fatorial inferior a 0,30 em todos os fatores dos modelos testados, ainda que tenha apresentado cargas mais expressivas nos fatores relacionados ao gerenciamento da impressão. Uma possivel explicação é que "resistir à tentação" não necessariamente ocorre enquanto um comportamento explicito. Da perspectiva egoísta, resistir à tentação implica um autocontrole das emoções e impulsos, sendo um comportamento socialmente aceitável. Da perspectiva moralista, esse autocontrole indica um respeito aos limites normativos estabelecidos pelo meio social do sujeito.

O modelo de quatro fatores (ou dois fatores e duas facetas) apresentou melhores índices de ajuste, mas resultando em uma interpretabilidade prejudicada dos padrões de cargas fatoriais. A versão com melhor interpretação dos dados foi o modelo de dois fatores, não obstante, como também aventado, o instrumento se mostrou capaz de captar os três fatores do modelo de Paulhus e Williams (1991). A dimensão gerenciamento da impressão permaneceu inalterada em relação ao modelo de dois fatores, e a dimensão autoengano se diferenciou entre autoengano positivo e negativo. Em relação ao modelo de quatro fatores, as dimensões referentes ao gerenciamento da imagem pouco se diferenciaram, conforme teoricamente esperado. Isso pode ter ocorrido pelo fato de a maioria dos itens focarem apenas no aspecto moralista da desejabilidade social e terem poucos itens do aspecto egoista, dificultando a identificação dessa faceta. Isso leva à hipótese de que valores melhores de índices de ajuste do modelo de quatro fatores ocorreu por conta da presença de mais parâmetros, o que resultou em menores resíduos (e.g., menor RMSEA). Assim, embora os valores estatísticos favoreçam o modelo de quatro fatores, a escala é mais indicada para avaliar os modelos de dois e três fatores por apresentarem melhores ajustes teóricos. 
Embora a solução de quatro fatores tenha sido aquela com o melhor ajuste aos dados, vale ressaltar que os três fatores testados não são necessariamente excludentes. A versão traduzida apresentou um valor de coeficiente alfa similar à versão original. As duas dimensões da escala apresentaram alfa entre 0,60 e 0,83. O valor elevado do alfa dos fatores nos três modelos testados indica uma alta correlação interitem, sugerindo que os itens avaliam um atributo similar. Basicamente, o modelo de três e quatro fatores, como discutido antes, consistem em uma subdivisão, em facetas, do modelo original de dois fatores. No caso dos resultados aqui encontrados, observou-se que a dimensão autoengano do modelo de dois fatores se dividiu entre os fatores dois e três do modelo de três fatores e quatro fatores, enquanto a dimensão gerenciamento da impressão teve os seus itens distribuídos entre os fatores um e quatro do modelo de quatro fatores. Ou seja, de fato, mesmo não tendo sido elaborado para captar as facetas dos modelos de Paulhus e Williams (1991) e de Paulhus e John (1998), a presente investigação sustenta o uso da escala IMSDS para essa finalidade, principalmente para a estrutura de três fatores. Pesquisadores podem usar a informação presente na Tabela 2 para o cômputo dos escores de cada fator (bem como para saber quais itens devem ter seus escores revertidos previamente). Mesmo assim, faz-se necessário correlacionar esses fatores com avaliações externas de comportamentos interpessoais, ajudando a estabelecer a validade externa das escalas (e.g., Pauls \& Stemmler, 2003).

O modelo fatorial reflexivo do instrumento significa que quanto mais alto o escore do indivíduo no instrumento, maior a probabilidade de apresentar respostas socialmente desejáveis. Os resultados preliminares aqui apresentados sugerem que a versão apresentada pelo IPIP possui boas propriedades psicométricas, sendo um recurso útil para pesquisadores envolvidos na avaliação do viés da desejabilidade social em adultos. Ainda assim, algumas limitações do estudo podem ser destacadas. Elas são o uso de apenas um único instrumento de desejabilidade social e o fato de ter uma composição amostral não aleatória que impossibilitam uma investigação mais consistente do atributo. Outro aspecto que ainda merece atenção é o teste de uma possivel estrutura hierárquica para o instrumento. Seria possivel que as dimensões moralista e egoísta se manifestassem enquanto fatores de segunda ordem, explicando esses quatro fatores encontrados na solução exploratória. A única dificuldade, que impediu o teste dessa hipótese neste estudo é que se trata de um modelo não identificado, que talvez só fosse testável no contexto de um modelo de equações estruturais mais complexo, incluindo outras variáveis. Nesse sentido, novos estudos devem ser conduzidos para buscar mais evidências de validade para o instrumento. Por fim, o estudo contribuiu para uma melhor compreensão dos diversos modelos explicativos de desejabilidade social, e, também, demostrou as possibilidades de uso da escala do IPIP para mensurar os modelos discutidos, sendo que as estruturas fatoriais apresentadas podem ser indicativas de como realizar o cômputo dos fatores.

\section{Referências}

Asparouhov, T., \& Muthén, B. (2009). Exploratory structural equation modeling. Structural Equation Modeling: A Multidisciplinary Journal, 16(3), 397-438. https://doi.org/10.1080/10705510903008204

Bäckström, M. (2008). Higher-order factors in a five-factor personality inventory and its relation to social desirability. International Journal of Psychology, 43(34), 370-371. https://doi.org/10.1027/1015-5759.23.2.63

Crowne, D., \& Marlowe, D. (1960). A new scale of social desirability independent of psychopathology. Journal Oj Consulting Psychology, 24(4), 349-354. https://doi.org/10.1037/ho047358

Ellingson, J. E. (2012). People fake only when they need to fake. In M. Ziegler, C. Maccann, \& R. D. Roberts (Eds.), New perspectives on faking in personality assessment (pp. 19-33). New York, NY: Oxford University Press, Inc. https://doi.org/10.1093/acprof:0so/9780195387476.003.0014

Leary, M. R., \& Bolino, M. C. (2017). An Actor-Perceiver Model of Impression Management in Organizations. In: Ferris, D. L., Johnson, R. E. \& Sedikides, C. (eds). The Self at Work: Fundamental Theory and Research. 253-267. https://doi.org/10.4324/9781315626543-11

Lorenzo-Seva, U., \& Ferrando, P. J. (2015). Factor: A computer program to fit the exploratory factor analysis model. Universitat Rovira i Virgili. (DOI INEXISTENTE) 
Bou Malham, P., \& Saucier, G. (2016). The conceptual link between social desirability and cultural normativity. International Journal of Psychology, 51(6), 474480. https://doi.org/10.1002/ijop.12261

Paulhus, D. L. (1984). Two-Component Models of Socially Desirable Responding. Personality Processes and Individual Differences, 46(3), 598-609. https://doi. org/10.1037/0022-3514.46.3.598

Paulhus, D. L. (2002). Socially desirable responding: the evolution of a construct. In H. I. Brown, D. N. Jackson, \& D. E. Wiley (Eds.), The role of constructs in psychological and educational measurement (pp. 49-69). Mahuah, NJ: Erlbaum. (DOI INEXISTENTE)

Paulhus, D. L. (2006). Comprehensive Inventory of Desirable Responding (CIDR). New Orleans. Recuperado de http://www2.psych.ubc.ca/ dpaulhus/ research/SDR/downloads/MEASURES/CIDR\%2O2. doc_(DOI INEXISTENTE)

Paulhus, D. L., \& John, O. P. (1998). Egoistic and moralistic biases in self-perception: The interplay of self-deceptive styles with basic traits and motives. Journal of Personality, 66(6), 1025-1060. https://doi. org/10.1111/1467-6494.00041

Paulhus, D. L., \& Reid, D. B. (1991). Enhancement and denial in socially desirable responding. Journal of Personality and Social Psychology, 60(2), 307-317. https://doi.org/10.1037/0022-3514.60.2.307

Pauls, C. A., \& Stemmler, G. (2003). Substance and bias in social desirability responding. Personality and Individual Differences, 35(2), 263-275. https://doi. org/10.1016/S0191-8869(02)00187-3

Timmerman, M. E., \& Lorenzo-Seva, U. (2011). Dimensionality assessment of ordered polytomous items with parallel analysis. Psychological Methods, 16(2), 209-220. https://doi.org/10.1037/a0023353

Trapnell, P. D., \& Paulhus, D. L. (2012). Agentic and communal values: Their scope and measurement. Journal of personality assessment, 94(1), 39-52. https://doi.org/10.1080/00223891.2011.627968

Vecchione, M., Alessandri, G., \& Barbaranelli, C. (2013). Measurement and application of egoistic and moralistic self-enhancements. International Journal of Selection and Assessment, 21(2), 170-182. https:// doi.org/10.1111/ijsa.12027

Schwardmann, Peter and van der Weele, Joel J. (2016). Deception and Self-Deception. Social Science Research Network (SSRN). https://doi.org/10.2139/ssrn.2734736

Ziegler, M. (2015). "F*** You, I Won't Do What You Told Me!" - Response Biases as Threats to Psychological Assessment. European Journal of Psychological Assessment, 31(3), 153-158. https://doi. org/10.1027/1015-5759/a000292

Zumbo, B. D., Gadermann, A. M., \& Zeisser, C. (2007). Ordinal Versions of Coefficients Alpha and Theta for Likert Rating Scales. Journal of Modern Applied Statistical Methods, 6(1), 21-29. https://doi.org/10.1107/ $\underline{\text { Sog074449009031205 }}$

\section{Endereço para correspondência:}

Ariela Raissa Lima Costa (Rua Waldemar César da Silveira, 105 - Jardim Cura D'ars CEP 13045510 Campinas - SP; +55 (11) 94042 8629; arielalima10@gmail.com2

\section{Autor I}

Nome: Ariela Raissa Lima Costa

Titulação Acadêmica: Mestre em Psicologia

Afiliação Institucional: Universidade São Francisco (USF)

\section{Autor II}

Nome: Nelson Hauck Filho

Titulação Acadêmica: Doutor em Psicologia

Afiliação Institucional: Universidade São Francisco (USF) 